\title{
Impact of trigger factors on clinical profile of migraine patients
}

\author{
Muddasir Sharief Banday ${ }^{1 *}$, Maqbool Wani², Bilal Ahmad Para ${ }^{3}$, Sabia Qureshi $^{4}$
}

${ }^{1}$ Department of Clinical Pharmacology, ${ }^{2}$ Department of Neurology, Sheri Kashmir Institute of Medical Sciences, Soura, J and K, India

${ }^{3}$ Department of Statistics, Government Degree College, Anantnag, J and K, India

${ }^{4}$ Division of Veterinary Microbiology and Immunology, FVSc and A. H., SKUAST-K, J and K, India

Received: 25 May 2020

Revised: 02 July 2020

Accepted: 03 July 2020

\section{*Correspondence:}

Dr. Muddasir Sharief Banday,

Email: banday.muddasir@gmail.com

Copyright: ( $)$ the author(s), publisher and licensee Medip Academy. This is an open-access article distributed under the terms of the Creative Commons Attribution Non-Commercial License, which permits unrestricted non-commercial use, distribution, and reproduction in any medium, provided the original work is properly cited.

\begin{abstract}
Background: Migraine is a primary headache disorder. The study was designed to provide a better understanding of the potential role of triggers in the cause of migraine and their impact on its clinical profile and treatment protocol.

Methods: A prospective study was conducted between June 2018 to May 2020 in 323 patients suffering from migraine in out-patient department of neurology. Patients were labelled as migraine on the basis of simplified diagnostic criteria for migraine. A structured questionnaire was used to interview patients about triggers and correlated with various clinical variables.

Results: All patients had migraine without aura with males 30 (9.3\%) and females 293 (90.7\%). Episodic migraine found more than chronic daily headache. Trigger factors were present in 234 (72.4\%) and absent in 89 (27.6\%) patients. Common triggers were hot climate, emotional stress, lack of sleep and fasting. Common foods to precipitate an attack are tomatoes, cheese and collard greens. Mean duration of headache in patients with trigger factors is $5.67 \pm 4.99$ years with a significant $p$ value $(p<0.02)$. Mean frequency of headache in trigger positive patients is $15.22 \pm 8.28$ (days/month). Clinical symptoms significant in trigger positive patients are nausea $(\mathrm{p}<0.0001)$ $(\mathrm{OR}=3.94 ; 95 \% \quad \mathrm{CI}=2.02-7.68)$, vomiting $\quad(\mathrm{p}=0.0001) \quad(\mathrm{OR}=2.62 ; 95 \% \quad \mathrm{CI}=1.50-4.59)$, photophobia $\quad(\mathrm{p}<0.0001)$ $(\mathrm{OR}=2.69 ; 95 \% \mathrm{CI}=1.56-4.64)$, phonophobia $(\mathrm{p}<0.0001)(\mathrm{OR}=5.16 ; 95 \% \mathrm{CI}=2.54-47)$, pulsating headache $(\mathrm{p}=0.006)$ $(\mathrm{OR}=2.09 ; 95 \% \mathrm{CI}=1.22-3.56)$, unilateral location $(\mathrm{p}<0.0001)(\mathrm{OR}=2.88 ; 95 \% \mathrm{CI}=1.74-4.77)$.

Conclusions: Triggers are not easily modifiable, and avoiding triggers may not be realistic. Healthy life style like exercise, adequate sleep, stress management and eating regularly may prevent triggers and transformation to chronification over time.
\end{abstract}

Keywords: Migraine, Headache, Trigger factors

\section{INTRODUCTION}

Migraine is a primary headache disorder. It is the second most common painful and incapacitating disorder in the world, afflicts approximately $15 \%$ of women and $6 \%$ of men over a one year period. ${ }^{1}$ It has a global prevalence of around one in seven people. ${ }^{2}$ According to global burden of disease study, it ranks as the seventh most common cause of disability worldwide, rising to the most common cause in the age of under $50 .^{3}$ Migraine is an important cause of reduced health related quality of life and has a significant and negative personal, societal and economic burden and is often underdiagnosed, misdiagnosed (e.g. in sinusitis) and undertreated in both primary and secondary care perhaps in part because there are no biological markers to confirm the diagnosis. ${ }^{4-8}$ The latest version of the international headache society's international classification of headache disorders 
ICHD-III (beta) (headache classification committee of the international headache society, 2013 classifies migraine as migraine without aura, migraine with aura, chronic migraine, complications of migraine, probable migraine, and episodic syndrome that may be associated with migraine. ${ }^{9}$ A simplified diagnostic criteria for migraine is quite reproducible which mentions as repeated attacks of headache lasting 4-17 hours in patients with a normal physical examination, no other reasonable cause for the headache and at least 2 of the these features as unilateral pain, throbbing pain, aggravation by movement, moderate or severe intensity plus at least one of the features like nausea/vomiting, photophobia and phonophobia. ${ }^{1}$

Clinical understanding and the definition of chronic migraine (CM) have evolved over time. In ICDH-3b, CM is broadly defined as migraine experienced greater than or equal to 15 days/month for more than 3 months with migrainous features, whereas in episodic migraine (EM) headache frequency lasts less than 15 days/month. ${ }^{9}$ Migraine attacks usually occur in response to identifiable triggers. ${ }^{10}$ Headache triggers have been defined as factors that alone or in combination, induce headache attacks in susceptible individuals. ${ }^{11}$ Triggers (also called precipitating factors) usually precede the attack by less than 48 h. Headache can be initiated or amplified by various triggers, including stress and negative emotions; hormonal factors for females; flicker, glare and eyestrain; noise; odours; hunger and consumption of certain foods and alcohol; weather; fatigue; and lack of sleep. The knowledge about migraine triggers is important for proper management of the patients. Trigger avoidance is sometimes referred to as 'headache hygiene'. There is paucity of studies in different ethnic populations of India to identify various triggering factors depending on diverse dietary habits. ${ }^{12}$ The study was designed with an objective to provide a better understanding of the potential role of triggers in the cause of migraine and to emphasize strategy of avoiding or coping with dietary and other migraine triggers before initiating long term drug therapy. In addition, migraine triggers were correlated with various clinical variables to understand the link between the trigger factors and headache in order to yield evidence based therapeutic approach in management protocol.

\section{METHODS}

A prospective study was conducted between June 2018 to May 2020 in 323 patients suffering from migraine in outpatient department of neurology in Sheri Kashmir Institute of Medical Sciences Soura, a 750 bedded multispecialty tertiary care teaching hospital. The study received an approval from Institutional Ethics Committee. Patients were labeled as migraines on the basis of simplified diagnostic criteria for migraine adapted from international headache society classification (headache classification committee of the international headache society 2013) patients were enrolled in the study after an informed written consent. A structured self-designed questionnaire was used to interview the patients clinically diagnosed as migraine for evaluation of endogenous (stress, negative emotions; hormonal factors for females) and exogenous (flicker, glare, eyestrain, noise, odour, hunger and consumption of certain foods and alcohol, weather, fatigue, and lack of sleep) triggers. Inclusion criteria included subjects aged 18-65 years with migraine without aura and migraine with aura. In exclusion criteria subjects with medication overuse headaches and other type of headaches were excluded. Detailed clinical history was taken in all patients and a clinical examination with specific reference to central nervous system (CNS) examination like Romberg's sign, tandem gait, drift of outstretched hands, finger-nose test, finger dexterity, binocular visual fields, to confrontation, eye movements, facial weakness, pupillary responses and Horner's syndrome, tendon reflexes and plantar responses and fundoscopy was carried out. The location of headache, total duration of migraine, its frequency per month and associated clinical presentation were also noted. Investigations like CT head and MRI brain was advised in some patients who did not respond to the initial treatment.

\section{Statistical analysis}

The data was analyzed by using statistical package for social sciences 22 program (SPSS Inc, Chicago, IL, USA). Fisher's exact test is used to identify the significant clinical symptoms with respect to trigger. The odds ratios (OR) and $95 \%$ confidence intervals (CI) were calculated to evaluate the measure of association between clinical variables and trigger factors. Descriptive statistics of clinical symptoms, triggering factors and other numeric variables is reported for an overview description of parameters. Independent sample t-test is employed, to assess the significance of age of patients (years), duration (years) and frequency of headache (days/month) between trigger positive and trigger negative patients. The level of significance was set at less than 0.05 for all analyses. Results were given as mean $\pm \mathrm{SD}$.

\section{RESULTS}

The study included 323 patients of migraine among which males were $30(9.3 \%)$ and females were 293 $(90.7 \%)$. The mean age of males is $38.80 \pm 17.53$ years and of females $35.38 \pm 13.29$ years, with a $p$ value of 0.307 . Most of them were in 21-30 years age group 26\%, followed by $31-40$ years $24.45 \%$. Table 1 summarizes the clinical symptoms, location, frequency of attacks and quality of headache in migraine patients. All the patients had migraine without aura and presented most commonly with phonophobia $88.2 \%$, nausea $87 \%$, photophobia $76.8 \%$, vomiting $56.9 \%$, light headedness $38.1 \%$, relief after vomiting $21 \%$, scalp tenderness $4.6 \%$ and vertigo $2.8 \%$. Patients presented with episodic migraine more frequently $62.2 \%$ than chronic daily headache $37.8 \%$. 
Table 1: Clinical symptoms, location of pain, frequency of attacks and quality of headache in migraine patients $(n=323)$.

\begin{tabular}{|c|c|c|c|}
\hline $\begin{array}{l}\text { Clinical } \\
\text { symptoms }\end{array}$ & Present/absent & Frequency & $\%$ \\
\hline \multirow{3}{*}{ Nausea } & No & 42 & 13 \\
\hline & Yes & 281 & 87 \\
\hline & Total & 323 & 100 \\
\hline \multirow{3}{*}{ Vomiting } & No & 119 & 43.1 \\
\hline & Yes & 157 & 56.9 \\
\hline & Total & 276 & 100 \\
\hline \multirow{3}{*}{$\begin{array}{l}\text { Relief after } \\
\text { vomiting }\end{array}$} & No & 252 & 79 \\
\hline & Yes & 67 & 21 \\
\hline & Total & 319 & 100 \\
\hline \multirow{3}{*}{$\begin{array}{l}\text { Light } \\
\text { headedness }\end{array}$} & No & 200 & 61.9 \\
\hline & Yes & 123 & 38.1 \\
\hline & Total & 323 & 100 \\
\hline \multirow{3}{*}{$\begin{array}{l}\text { Scalp } \\
\text { tenderness }\end{array}$} & No & 308 & 95.4 \\
\hline & Yes & 15 & 4.6 \\
\hline & Total & 323 & 100 \\
\hline \multirow{3}{*}{ Photophobia } & No & 75 & 23.2 \\
\hline & Yes & 248 & 76.8 \\
\hline & Total & 323 & 100 \\
\hline \multirow{3}{*}{ Phonophobia } & No & 38 & 11.8 \\
\hline & Yes & 284 & 88.2 \\
\hline & Total & 322 & 100 \\
\hline \multirow{3}{*}{ Vertigo } & No & 314 & 97.2 \\
\hline & Yes & 9 & 2.8 \\
\hline & Total & 323 & 100 \\
\hline \multirow{3}{*}{$\begin{array}{l}\text { Frequency of } \\
\text { headache } \\
\text { (days/month) }\end{array}$} & $\begin{array}{l}\text { Episodic migraine } \\
(\mathrm{EM})\end{array}$ & 201 & 62.2 \\
\hline & $\begin{array}{l}\text { Chronic daily } \\
\text { headache (CDH) }\end{array}$ & 122 & 37.8 \\
\hline & Total & 323 & 100 \\
\hline \multirow{3}{*}{$\begin{array}{l}\text { Pulsating } \\
\text { quality } \\
\text { headache }\end{array}$} & No & 95 & 30.7 \\
\hline & Yes & 214 & 69.3 \\
\hline & Total & 309 & 100 \\
\hline \multirow{3}{*}{$\begin{array}{l}\text { Unilateral/he } \\
\text { mi-cranial }\end{array}$} & No & 132 & 40.9 \\
\hline & Yes & 191 & 59.1 \\
\hline & Total & 323 & 100 \\
\hline \multirow{3}{*}{$\begin{array}{l}\text { Global/holo- } \\
\text { cranial }\end{array}$} & No & 201 & 62.2 \\
\hline & Yes & 122 & 37.8 \\
\hline & Total & 323 & 100 \\
\hline \multirow{3}{*}{$\begin{array}{l}\text { Occipital/nuc } \\
\text { hal }\end{array}$} & No & 291 & 90.1 \\
\hline & Yes & 32 & 9.9 \\
\hline & Total & 323 & 100 \\
\hline \multirow{3}{*}{ Bilateral } & No & 318 & 98.5 \\
\hline & Yes & 5 & 1.5 \\
\hline & Total & 323 & 100 \\
\hline \multirow{3}{*}{$\begin{array}{l}\text { Bitemporal } \\
\text { with } \\
\text { supraorbital }\end{array}$} & No & 307 & 95 \\
\hline & Yes & 16 & 5 \\
\hline & Total & 323 & 100 \\
\hline \multirow{3}{*}{ Frontal } & No & 305 & 94.4 \\
\hline & Yes & 18 & 5.6 \\
\hline & Total & 323 & 100 \\
\hline
\end{tabular}

Classical pulsating headache was found in $69.3 \%$ of patients while others presented with pain of mild to moderate severity with no pulsating quality. With regards to location of headache, unilateral was found in $59.1 \%$ of patients followed by holo-cranial $37.8 \%$, occipital $9.9 \%$, frontal $5.6 \%$, bitemporal with supraorbital $5 \%$ and bilateral $1.5 \%$. The mean duration of headache was $5.3 \pm 4.7$ years and the frequency of attacks was $14.74 \pm 8.2$ (days/month).

Trigger factors were present in $234(72.4 \%)$ and absent in $89(27.6 \%)$ patients as shown in (Figure 1$)$.

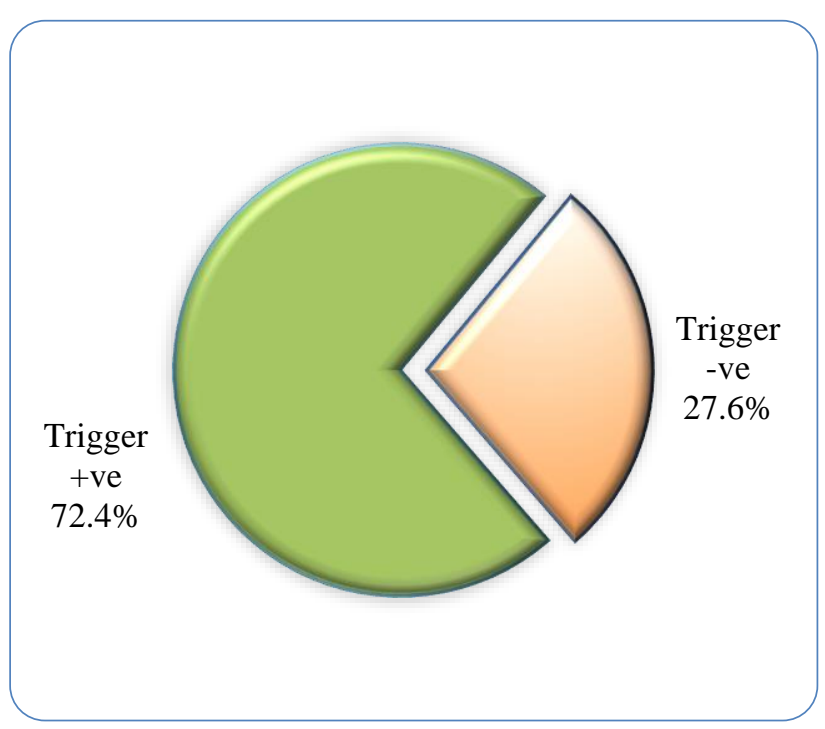

Figure 1: Percentage of presence and absence of trigger factors in migraine patients.

$-\mathrm{ve}=$ trigger absent, $+\mathrm{ve}=$ trigger present.

Table 2: Trigger factors in migraine patients $(n=323)$.

\begin{tabular}{|lll|}
\hline Trigger factors & $\begin{array}{l}\text { Number of } \\
\text { patients }\end{array}$ & $\%$ \\
\hline Tomatoes & 35 & 10.8 \\
\hline Cheese & 24 & 7.4 \\
\hline Onions & 2 & 0.6 \\
\hline $\begin{array}{l}\text { Collard greens/Kale } \\
\text { (Haak) }\end{array}$ & 21 & 6.5 \\
\hline Hot climate & 118 & 36.5 \\
\hline Fasting & 78 & 24.1 \\
\hline $\begin{array}{l}\text { Lack of sleep/sleep } \\
\text { deprivation }\end{array}$ & 86 & 26.6 \\
\hline Emotional stress/anxiety & 118 & 36.5 \\
\hline Spicy junk food & 4 & 1.2 \\
\hline Exposure to cold & 3 & 0.9 \\
\hline Pickles & 4 & 1.2 \\
\hline Turnips and radish & 5 & 1.5 \\
\hline Pulses and beans & 5 & 1.5 \\
\hline Workload & 2 & 0.6 \\
\hline Menstrual cycle & 3 & 0.9 \\
\hline Total & 508 & 157.3 \\
\hline
\end{tabular}


Most common trigger factors observed in our ethnic population are depicted in (Table 2). The common food items which are seen to precipitate an attack are tomatoes $35(10.8 \%)$, cheese $24(7.4 \%)$, collard greens/kale (Haakh) 21 (6.5\%), (in Kashmir valley collard greens popularly known as Haakh in local parlance are included in most of the meals and both the leaves and roots are consumed. It belongs to species Brassica oleracea) turnips and radish $5(1.5 \%)$, pulses and beans $5(1.5 \%)$, pickles $4(1.2 \%)$ and onions $2(0.6 \%)$.

Majority of patients had multiple trigger factors varying from nil to maximum of seven. Figure 2, depicts the percentage of patients with none, one or more than one trigger factors responsible for precipitating an attack.

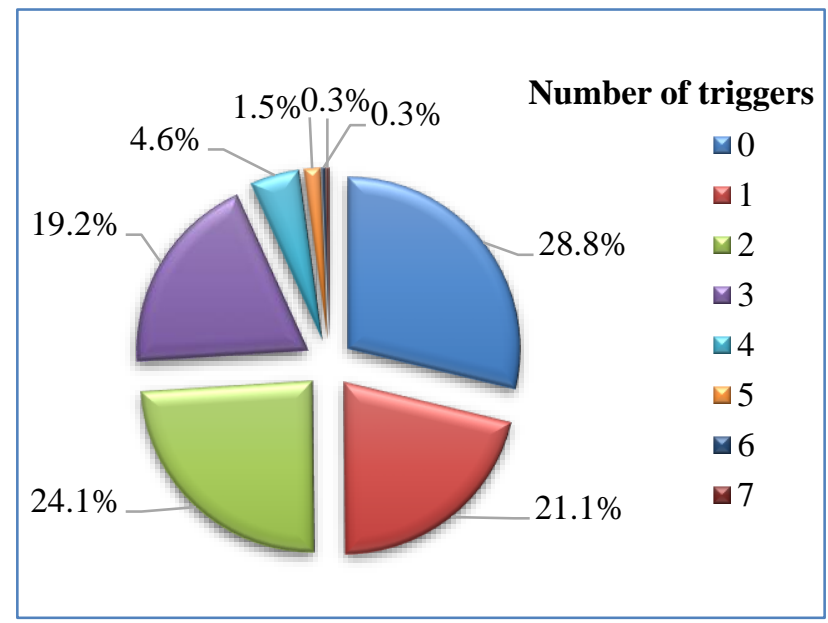

Figure 2: Percentage of zero to seven trigger factors in migraine patients.
Table 3: Relationship of age, duration of headache and frequency of attack in migraine patients with and without trigger.

\begin{tabular}{|c|c|c|c|c|}
\hline Trigger factor & & $\mathbf{N}$ & $\begin{array}{l}\text { Mean } \\
\pm \text { SD }\end{array}$ & P value \\
\hline \multirow{2}{*}{$\begin{array}{l}\text { Age of } \\
\text { subjects } \\
\text { (years) }\end{array}$} & $\begin{array}{l}\text { Trigger } \\
-\mathrm{ve}\end{array}$ & 82 & $\begin{array}{l}35.659 \pm \\
15.736\end{array}$ & \multirow{2}{*}{0.974} \\
\hline & $\begin{array}{l}\text { Trigger } \\
+\mathrm{ve}\end{array}$ & 233 & $\begin{array}{l}35.717 \pm \\
13.028\end{array}$ & \\
\hline \multirow{2}{*}{$\begin{array}{l}\text { Duration of } \\
\text { headache } \\
\text { (years) }\end{array}$} & $\begin{array}{l}\text { Trigger } \\
- \text { ve }\end{array}$ & 78 & $\begin{array}{l}4.397 \pm \\
3.822\end{array}$ & \multirow{2}{*}{0.020} \\
\hline & $\begin{array}{l}\text { Trigger } \\
+\mathrm{ve}\end{array}$ & 232 & $\begin{array}{l}5.672 \pm \\
4.998\end{array}$ & \\
\hline \multirow{2}{*}{$\begin{array}{l}\text { Frequency of } \\
\text { headache } \\
\text { (days/month) }\end{array}$} & $\begin{array}{l}\text { Trigger } \\
-\mathrm{ve}\end{array}$ & 79 & $\begin{array}{l}13.342 \pm \\
8.184\end{array}$ & \multirow{2}{*}{0.081} \\
\hline & $\begin{array}{l}\text { Trigger } \\
+ \text { ve }\end{array}$ & 231 & $\begin{array}{l}15.225 \pm \\
8.288\end{array}$ & \\
\hline
\end{tabular}

*Results are given as mean $\pm \mathrm{SD}$. Independent $\mathrm{T}$-test was used to compare groups with significance of $\mathrm{p}<0.05,-\mathrm{ve}=$ absent, $+\mathrm{ve}=$ present, $\mathrm{N}$ frequency.

Table 3 represents the relationship between mean age of patients, duration of headache and frequency of attack with patients presenting with or without trigger factor in the neurology clinic. The mean duration of headache in patients who present with a history of trigger factor or factors is $5.67 \pm 4.99$ years with a significant $p$ value $(p<0.02)$. The mean frequency of headache in trigger positive patients is $15.22 \pm 8.28$ (days/month) and the $p$ value is 0.081 . Table 4 summarizes the correlation between the clinical symptoms, location of pain, frequency of attacks and quality of headache with the trigger factors associated with migraine patients.

Table 4: Correlation of clinical symptoms, location of pain and frequency of attacks and nature of pain in patients with and without trigger.

\begin{tabular}{|c|c|c|c|c|c|c|c|c|}
\hline \multirow{2}{*}{\multicolumn{2}{|c|}{ Clinical symptoms }} & \multicolumn{2}{|c|}{ Triggering factor } & \multirow[b]{2}{*}{ Total } & \multirow[b]{2}{*}{ P value } & \multirow{2}{*}{$\begin{array}{l}\text { Odds } \\
\text { ratio } \\
\text { (OR) }\end{array}$} & \multicolumn{2}{|c|}{$95 \%$ Confidence interval } \\
\hline & & $\begin{array}{l}\text { Trigger } \\
\text {-ve }\end{array}$ & $\begin{array}{l}\text { Trigger } \\
+ \text { ve }\end{array}$ & & & & Lower limit & Upper limit \\
\hline \multirow{4}{*}{ Nausea } & \multirow{2}{*}{ No } & 23 & 19 & 42 & \multirow{6}{*}{$<0.0001$} & \multirow{6}{*}{3.943} & \multirow{6}{*}{2.023} & \multirow{6}{*}{7.685} \\
\hline & & $54.8 \%$ & $45.2 \%$ & $100.0 \%$ & & & & \\
\hline & \multirow{2}{*}{ Yes } & 66 & 215 & 281 & & & & \\
\hline & & $23.5 \%$ & $76.5 \%$ & $100.0 \%$ & & & & \\
\hline \multirow{2}{*}{ Total } & & 89 & 234 & 323 & & & & \\
\hline & & $27.6 \%$ & $72.4 \%$ & $100.0 \%$ & & & & \\
\hline \multirow{4}{*}{ Vomiting } & \multirow{2}{*}{ No } & 42 & 77 & 119 & \multirow{6}{*}{0.001} & \multirow{6}{*}{2.626} & \multirow{6}{*}{1.501} & \multirow{6}{*}{4.596} \\
\hline & & $35.3 \%$ & $64.7 \%$ & $100.0 \%$ & & & & \\
\hline & \multirow{2}{*}{ Yes } & 27 & 130 & 157 & & & & \\
\hline & & $17.2 \%$ & $82.8 \%$ & $100.0 \%$ & & & & \\
\hline \multirow{2}{*}{ Total } & & 69 & 207 & 276 & & & & \\
\hline & & $25.0 \%$ & $75.0 \%$ & $100.0 \%$ & & & & \\
\hline \multirow{4}{*}{$\begin{array}{l}\text { Relief after } \\
\text { vomiting }\end{array}$} & \multirow{2}{*}{ No } & 74 & 178 & 252 & \multirow{6}{*}{0.218} & \multirow{6}{*}{1.574} & \multirow{6}{*}{0.823} & \multirow{6}{*}{3.010} \\
\hline & & $29.4 \%$ & $70.6 \%$ & $100.0 \%$ & & & & \\
\hline & \multirow{2}{*}{ Yes } & 14 & 53 & 67 & & & & \\
\hline & & $20.9 \%$ & $79.1 \%$ & $100.0 \%$ & & & & \\
\hline \multirow{2}{*}{ Total } & & 88 & 231 & 319 & & & & \\
\hline & & $27.6 \%$ & $72.4 \%$ & $100.0 \%$ & & & & \\
\hline
\end{tabular}

Continued. 


\begin{tabular}{|c|c|c|c|c|c|c|c|c|}
\hline \multirow{2}{*}{\multicolumn{2}{|c|}{ Clinical symptoms }} & \multicolumn{2}{|c|}{ Triggering factor } & \multirow[b]{2}{*}{ Total } & \multirow[b]{2}{*}{ P value } & \multirow{2}{*}{$\begin{array}{l}\text { Odds } \\
\text { ratio } \\
\text { (OR) }\end{array}$} & \multicolumn{2}{|c|}{$95 \%$ Confidence interval } \\
\hline & & $\begin{array}{l}\text { Trigger } \\
\text {-ve }\end{array}$ & $\begin{array}{l}\text { Trigger } \\
\text { +ve }\end{array}$ & & & & Lower limit & Upper limit \\
\hline \multirow{4}{*}{$\begin{array}{l}\text { Light } \\
\text { headedness }\end{array}$} & \multirow{2}{*}{ No } & 57 & 143 & 200 & \multirow{6}{*}{0.628} & \multirow{6}{*}{1.134} & \multirow{6}{*}{0.683} & \multirow{6}{*}{1.881} \\
\hline & & $28.5 \%$ & $71.5 \%$ & $100.0 \%$ & & & & \\
\hline & \multirow{2}{*}{ Yes } & 32 & 91 & 123 & & & & \\
\hline & & $26.0 \%$ & $74.0 \%$ & $100.0 \%$ & & & & \\
\hline \multirow{2}{*}{ Total } & & 89 & 234 & 323 & & & & \\
\hline & & $27.6 \%$ & $72.4 \%$ & $100.0 \%$ & & & & \\
\hline \multirow{4}{*}{ Scalp tenderness } & \multirow{2}{*}{ No } & 86 & 222 & 308 & & & & \\
\hline & & $27.9 \%$ & $72.1 \%$ & $100.0 \%$ & & & & \\
\hline & \multirow{2}{*}{ Yes } & 3 & 12 & 15 & & & & \\
\hline & & $20.0 \%$ & $80.0 \%$ & $100.0 \%$ & 0.168 & 1.550 & 0.421 & 5.626 \\
\hline & & 89 & 234 & 323 & & & & \\
\hline Total & & $27.6 \%$ & $72.4 \%$ & $100.0 \%$ & & & & \\
\hline & $\mathrm{No}$ & 33 & 42 & 75 & & & & \\
\hline & No & $44.0 \%$ & $56.0 \%$ & $100.0 \%$ & & & & \\
\hline Photophobia & & 56 & 192 & 248 & $<\cap 0 \cap 01$ & 2604 & 1562 & 1644 \\
\hline & Yes & $22.6 \%$ & $77.4 \%$ & $100.0 \%$ & $<0.0001$ & 2.694 & 1.563 & 4.644 \\
\hline Totol & & 89 & 234 & 323 & & & & \\
\hline Total & & $27.6 \%$ & $72.4 \%$ & $100.0 \%$ & & & & \\
\hline & $\mathrm{No}$ & 23 & 15 & 38 & & & & \\
\hline Phononhohia & No & $60.5 \%$ & $39.5 \%$ & $100.0 \%$ & & & & \\
\hline Pnonopnobla & Yes & 65 & 219 & 284 & $<00001$ & 5166 & 5,548 & 10476 \\
\hline & Yes & $22.9 \%$ & $77.1 \%$ & $100.0 \%$ & $<0.0001$ & 5.100 & 2.548 & $10.4 / 0$ \\
\hline Total & & 88 & 234 & 322 & & & & \\
\hline Iotal & & $27.3 \%$ & $72.7 \%$ & $100.0 \%$ & & & & \\
\hline & $\mathrm{FM}$ & 55 & 138 & 193 & & & & \\
\hline Frequency of & ElM & $28.5 \%$ & $71.5 \%$ & $100.0 \%$ & & & & \\
\hline (days/month) & $\mathrm{CD}$ & 24 & 93 & 117 & & & & \\
\hline & $\mathrm{H}$ & $20.5 \%$ & $79.5 \%$ & $100.0 \%$ & 0.139 & 1.544 & 0.894 & 2.668 \\
\hline & & 79 & 231 & 310 & & & & \\
\hline Total & & $25.5 \%$ & $74.5 \%$ & $100.0 \%$ & & & & \\
\hline & & 34 & 61 & 95 & & & & \\
\hline Pulsating & No & $35.8 \%$ & $64.2 \%$ & $100.0 \%$ & & & & \\
\hline headache & Yes & 45 & 169 & 214 & 0006 & 2003 & 1228 & 3567 \\
\hline & Yes & $21.0 \%$ & $79.0 \%$ & $100.0 \%$ & 0.006 & 2.093 & 1.228 & $3.56 /$ \\
\hline Total & & 79 & 230 & 309 & & & & \\
\hline Total & & $25.6 \%$ & $74.4 \%$ & $100.0 \%$ & & & & \\
\hline & $\mathrm{No}$ & 53 & 79 & 132 & & & & \\
\hline Unilateral/hemi- & No & $40.2 \%$ & $59.8 \%$ & $100.0 \%$ & & & & \\
\hline cranial & Yes & 36 & 155 & 191 & $<00001$ & 2880 & 1747 & 4775 \\
\hline & Yes & $18.8 \%$ & $81.2 \%$ & $100.0 \%$ & $<0.0001$ & 2.889 & $1.14 /$ & $4 . / 15$ \\
\hline Totol & & 89 & 234 & 323 & & & & \\
\hline Total & & $27.6 \%$ & $72.4 \%$ & $100.0 \%$ & & & & \\
\hline & $\mathrm{No}$ & 48 & 153 & 201 & & & & \\
\hline Global/holo- & No & $23.9 \%$ & $76.1 \%$ & $100.0 \%$ & & & & \\
\hline cranial & & 41 & 81 & 122 & 0073 & 0620 & 0377 & \\
\hline & Yes & $33.6 \%$ & $66.4 \%$ & $100.0 \%$ & 0.073 & 0.620 & $0.3 / 7$ & 1.018 \\
\hline Totol & & 89 & 234 & 323 & & & & \\
\hline Total & & $27.6 \%$ & $72.4 \%$ & $100.0 \%$ & & & & \\
\hline & & 82 & 209 & 291 & & & 0.583 & \\
\hline Occipital/nuchal & No & $28.2 \%$ & $71.8 \%$ & $100.0 \%$ & & & & \\
\hline headache & & 7 & 25 & 32 & & & & \\
\hline & Yes & $21.9 \%$ & $78.1 \%$ & $100.0 \%$ & 0.536 & 1.401 & & 3.365 \\
\hline & & 89 & 234 & 323 & & & & \\
\hline & & $27.6 \%$ & $72.4 \%$ & $100.0 \%$ & & & & \\
\hline
\end{tabular}

Continued. 


\begin{tabular}{|c|c|c|c|c|c|c|c|c|}
\hline \multirow{2}{*}{\multicolumn{2}{|c|}{ Clinical symptoms }} & \multicolumn{2}{|c|}{ Triggering factor } & \multirow[b]{2}{*}{ Total } & \multirow[b]{2}{*}{$P$ value } & \multirow{2}{*}{$\begin{array}{l}\text { Odds } \\
\text { ratio } \\
\text { (OR) }\end{array}$} & \multicolumn{2}{|c|}{ 95\% Confidence interval } \\
\hline & & $\begin{array}{l}\text { Trigger } \\
\text {-ve }\end{array}$ & $\begin{array}{l}\text { Trigger } \\
+ \text { ve }\end{array}$ & & & & Lower limit & Upper limit \\
\hline \multirow{4}{*}{$\begin{array}{l}\text { Bilateral } \\
\text { headache }\end{array}$} & \multirow{2}{*}{ No } & 88 & 230 & 318 & \multirow{6}{*}{0.703} & \multirow{6}{*}{1.530} & \multirow{6}{*}{0.169} & \multirow{6}{*}{13.882} \\
\hline & & $27.7 \%$ & $72.3 \%$ & $100.0 \%$ & & & & \\
\hline & \multirow{2}{*}{ Yes } & 1 & 4 & 5 & & & & \\
\hline & & $20.0 \%$ & $80.0 \%$ & $100.0 \%$ & & & & \\
\hline \multirow{2}{*}{\multicolumn{2}{|c|}{ Total }} & 89 & 234 & 323 & & & & \\
\hline & & $27.6 \%$ & $72.4 \%$ & $100.0 \%$ & & & & \\
\hline \multirow{4}{*}{$\begin{array}{l}\text { Bitemporal with } \\
\text { supraorbital }\end{array}$} & \multirow{2}{*}{ No } & 85 & 222 & 307 & \multirow{6}{*}{0.815} & \multirow{6}{*}{1.149} & \multirow{6}{*}{0.360} & \multirow{6}{*}{3.660} \\
\hline & & $27.7 \%$ & $72.3 \%$ & $100.0 \%$ & & & & \\
\hline & \multirow{2}{*}{ Yes } & 4 & 12 & 16 & & & & \\
\hline & & $25.0 \%$ & $75.0 \%$ & $100.0 \%$ & & & & \\
\hline \multirow{2}{*}{ Total } & & 89 & 234 & 323 & & & & \\
\hline & & $27.6 \%$ & $72.4 \%$ & $100.0 \%$ & & & & \\
\hline \multirow{4}{*}{$\begin{array}{l}\text { Frontal } \\
\text { headache }\end{array}$} & \multirow{2}{*}{ No } & 84 & 221 & 305 & \multirow{7}{*}{0.983} & \multirow{7}{*}{0.988} & \multirow{7}{*}{0.342} & \multirow{7}{*}{2.857} \\
\hline & & $27.5 \%$ & $72.5 \%$ & $100.0 \%$ & & & & \\
\hline & \multirow{2}{*}{ Yes } & 5 & 13 & 18 & & & & \\
\hline & & $27.8 \%$ & $72.2 \%$ & $100.0 \%$ & & & & \\
\hline \multirow{3}{*}{ Total } & & 89 & 234 & 323 & & & & \\
\hline & & $27.6 \%$ & $72.4 \%$ & $100.0 \%$ & & & & \\
\hline & & $27.6 \%$ & $72.4 \%$ & $100.0 \%$ & & & & \\
\hline
\end{tabular}

$\mathrm{ve}=$ trigger absent, $+\mathrm{ve}=$ trigger present, $\mathrm{EM}$ : episodic migraine, $\mathrm{CDH}$ : chronic daily headache.

Nausea was present in 281 patients out of which 215 $(76.5 \%)$ presented with history of one or more trigger factors with a $\mathrm{p}$ value of $(\mathrm{p}<0.0001),(\mathrm{OR}=3.94 ; 95 \%$ $\mathrm{CI}=2.02-7.68)$. Vomiting in $130(82.8 \%)$ patients with trigger factors with a $\mathrm{p}$ value of $(\mathrm{p}=0.0001),(\mathrm{OR}=2.62$ 95\% CI=1.50-4.59). Photophobia was seen in 192 $(77.4 \%)$ patients with trigger with a $\mathrm{p}$ value of ( $\mathrm{p}<0.0001),(\mathrm{OR}=2.69 ; 95 \% \mathrm{CI}=1.56-4.64)$. Phonophobia in $219(77.1 \%)$ patients with trigger with a $\mathrm{p}$ value of $(\mathrm{p}<0.0001),(\mathrm{OR}=5.16 ; 95 \% \mathrm{CI}=2.54-10.47)$. Pulsating nature of headache was seen in $169(79.0 \%)$ patients with history of one or more trigger factors, with a $\mathrm{p}$ value of $(\mathrm{p}=0.006,(\mathrm{OR}=2.09 ; 95 \% \mathrm{CI}=1.22-3.56)$. The pain was most commonly perceived as unilateral/hemi-cranial in $155(81.2 \%)$ patients with triggers and a $\mathrm{p}$ value $(\mathrm{p}<0.0001), \quad(\mathrm{OR}=2.88 ; \quad 95 \% \quad \mathrm{CI}=1.74-4.77) . \quad$ Other clinical symptoms, frequency of headache (days/month) and other locations of headache did not show significant values with respect to presence or absence of different trigger factors.

\section{DISCUSSION}

The present study observed that in our ethnic population all the patients had migraine without aura and most frequently reported symptoms were phonophobia, nausea, photophobia, vomiting, lightheadedness, relief after vomiting, scalp tenderness and vertigo. Pulsatile pain was found in $69.3 \%$ and unilateral pain in $59.1 \%$ of migraineurs (Table 1). Similar findings have been observed in a US population based survey conducted by Lipton et al which shows photophobia (80\% of migraineures), phonophobia (76\% of migraineures), nausea $(73 \%)$, vomiting $(29 \%)$, pulsatile pain $(85 \%$ of migraineures) and unilateral pain in $59 \% .{ }^{13}$ Our study shows trigger factors were present in $72.4 \%$ of patients. The commonest trigger factors which have been identified are hot climate, emotional stress/anxiety, lack of sleep/sleep deprivation and fasting. Common food items identified as triggers were tomatoes, cheese and collard greens (Table 2). Majority of patients had multiple trigger factors, with no trigger in $28.8 \%$ of migraineures, one in $21.1 \%$, two in $24.1 \%$, three in $19.2 \%$, four in $4.6 \%$, five in $1.5 \%$, six in $0.3 \%$ and seven in $0.3 \%$ of patients. According to study conducted by Martin et al, anxiety emerged as one of the most common triggering factors of migraine. ${ }^{14}$ Predominantly individuals react to the headache in the form of anxiousness, related to cause of headache, how to manage it and it impact on them. The anxiety reaction creates negative feedback loops, thereby increasing the perception of pain and exacerbates anxiety trigger factor. A clinical study by Kelman found that $76 \%$ of migraineures responded affirmatively when asked whether they had triggers for migraine attacks. ${ }^{15}$ Moreover, Berg et al, indicated that migraineures reported a median of 3 triggers with a range from 1 to 12. ${ }^{16}$ These studies are consistent with our study where triggers range from nil to 7 . Fasting was one of the common triggers in our study $24.1 \%$. In our ethnic population fasting is commonly observed as a religious obligation especially in the holy month of Ramdhan. Missing a meal due to occupational reasons is yet another reason for fasting. Similar findings have been reported in a study conducted by Yadav et al, in India. ${ }^{17}$ Stress hormone release and hypoglycemia has been implicated to trigger a migraine attack due to fasting. ${ }^{18}$ Water deprivation has also been attributed to trigger migraine attack. ${ }^{19}$ Diet can play an important role in the precipitation of headaches in children and adolescents 
with migraine. The list of foods, beverages, and additives that trigger migraine includes cheese, chocolate, citrus fruits, hot dogs, monosodium glutamate, aspartame, fatty foods, ice cream, caffeine withdrawal, and alcoholic drinks, especially red wine and beer. ${ }^{18,20}$ In our study the dietary triggers which have been implicated to trigger headaches are tomatoes, cheese, collard greens, pickles and junk food. A study carried by Spierings et al reported the factors indicated most frequently as precipitating headache by the patients with migraine were stress/tension $84 \%$, not eating on time/fasting $82 \%$, fatigue $79 \%$, and lack of sleep $74 \% .^{21}$ These studies are consistent with our study except for certain dietary items which are native to our ethnic population. In current study clinical symptoms have been correlated in patients in without precipitating factors for migraine attacks. Very little studies have been so far conducted where the influence of trigger factors on clinical presentation has been studied. In our study clinical symptoms like nausea, vomiting, photophobia, phonophobia, pulsatile and unilateral location of headache show significant p-values in patients who have established precipitating factors. The study has paved way for further research with a robust data to establish a link between intensity and degree of occurrence of clinical symptoms and presence of trigger factors in patients of migraine.

\section{CONCLUSION}

Both EM and CDH are significant health issues, cause much suffering, adversely effects an individual's functional capacity and has a high societal cost. Headaches are precipitated by triggers and advice to avoid triggers is a standard feature of clinical management. But currently the concept of "coping with triggers should replace the idea of avoiding triggers". A behavioral management involves graded exposure to desensitize the headache sufferers to some triggers, whilst reserving avoidance only for selected ones with an increased potential for eliciting headache with a secondary benefit of less restricted lifestyle, encouragement of a well-balanced diet and enhanced selfefficacy for preventing headache. This may be preferable to long-term prophylactic drug treatment with attending adverse reaction.

\section{Funding: No funding sources}

Conflict of interest: None declared

Ethical approval: The study was approved by the Institutional Ethics Committee

\section{REFERENCES}

1. Peter JG, Neil HR. Migraine and other Primary Headache Disorders In: Dennis LK, Anthony SF, Stephen LH, Dan LL, Larry J, Joseph L, eds Harrison's Principles of Internal Medicine. Mc Graw Hill; 2016:2586-98.

2. Steiner TJ, Stovner LJ, Birbeck GL. Migraine: the seventh disabler. J Headache Pain. 2013;14:1.
3. Steiner TJ, Stovner LJ, Vos T. GBD 2015: migraine is the third cause of disability in under 50s. J Headache Pain. 2016;17(1):104.

4. Lipton RB, Bigal ME, Diamond M, Freitag F, Reed ML, Stewart WF. Migraine prevalence, disease burden, and the need for preventive therapy. Neurology. 2007;68:343-9.

5. Hawkins K, Wang S, Rupnow M. Direct cost burden among insured US employees with migraine. Headache. 2008;48:553-63.

6. Vos T, Flaxman AD, Naghavi M. Years lived with disability (YLD) for 1160 sequelae of 289 diseases and injuries 1990-2010: a systematic analysis for the Global Burden of Disease Study 2010. Lancet. 2012;380:2163-96.

7. Blumenfeld AM, Varon SF, Wilcox TK. Disability, HR QoL and resource use among chronic and episodic migraineures: results from the International Burden of Migraine Study (IBMS). Cephalalgia. 2011;31:301-15.

8. Schreiber CP, Hutchinson S, Webster CJ, Ames M, Richardson MS, Powers C. Prevalence of migraine in patients with a history of self-reported or physician diagnosed "sinus" headache. Arch Intern Med. 2004;44(9):856-64.

9. Headache Classification Committee of the International Headache society (IHS); the international Classification of Headache Disorders, 3rd edition (beta version). Cephalalgia. 2013;33(9):629-808.

10. Kelman L. The triggers or precipitants of the acute migraine attack. Cephalalgia. 2007;27:394-402.

11. Zagami AS, Bahra A. Symptomatology of migraines without aura. In J P Olesen J. N. Goadsby M. Ramadan P. T felt-Hansen, and K. M. A. Welch (Eds.), The headaches (pp. 399-405)., (3rd. ed.). Philadelphia: Lippincott Williams and Wilkins; 2006.

12. Rama KY, Jayantee K, Usha KM. A study of triggers of migraine in India. Pain Med. 2010;11:44-7.

13. Lipton RB, Stewart WF, Diamond S, Diamond ML, Reed M. Prevalence and Burden of Migraine in US: Data From the American Migraine study II. Headache. 2001;41:646-57.

14. Martin PR, Milech D, Nathan PR. Towards a functional model of chronic headaches. Headache. 1993;33:461-70.

15. Kelman L. The triggers or precipitants of the acute migraine attack. Cephalalgia. 2007;27:394-402.

16. Bergh VD, Amery WK, Waelkens J. Trigger factors in migraine: a study conducted by the Belgian migraine society. Headache. 1987;27:191-6.

17. Yadav RK, Kalita J, Misra UK. A Study of Triggers of Migraine in India. Pain Med. 2010;11:44-7.

18. Millichap JG, Yee MM. The diet factor in pediatric and adolescent migraine. Pediatr Neurol. 2003;28:9-15.

19. Blau JN. Water deprivation: a new migraine precipitant. Headache. 2005;45:757-9.

20. Migraine HE. In: Lessof MH, ed. Clinical reactions to food. Chichester: John Wiley; 1983: 155-180. 
21. Spierings ELH, Ranke AH, Honkoop PC Precipitating and Aggravating Factors of Migraine versus Tension type Headache. Headache. 2001;41:554-8.

Cite this article as: Banday MS, Wani M, Para BA, Qureshi S. Impact of trigger factors on clinical profile of migraine patients. Int $\mathrm{J}$ Basic Clin Pharmacol 2020;9:1191-8. 